Research Article

\title{
Research on a Grey Prediction Model of Population Growth Based on a Logistic Approach
}

\author{
Mingyu Tong (iD, Zou Yan, and Liu Chao \\ School of Economics \& Management, Chongqing Normal University, Chongqing 401131, China \\ Correspondence should be addressed to Mingyu Tong; tongmy@cqnu.edu.cn
}

Received 27 April 2020; Revised 4 September 2020; Accepted 26 September 2020; Published 15 October 2020

Academic Editor: Dorota Mozyrska

Copyright ( $\odot 2020$ Mingyu Tong et al. This is an open access article distributed under the Creative Commons Attribution License, which permits unrestricted use, distribution, and reproduction in any medium, provided the original work is properly cited.

\begin{abstract}
The classical population growth models include the Malthus population growth model and the logistic population growth model, each of which has its advantages and disadvantages. To address the disadvantages of the two models, this paper establishes a grey logistic population growth prediction model, based on the modeling mechanism of the grey prediction model and the characteristics of the logistic model, which uses the least-squares method to estimate the maximum population capacity. In accordance with the data characteristics of population growth, the weakening buffer operator is used to establish the weakening buffer operator grey logistic population growth prediction model, which improves its accuracy, thus improving the classic population prediction model. Four actual case datasets are used simultaneously, and the two classical grey prediction models are compared. The results of the six evaluation indicators show that the effects of the new model demonstrate obvious advantages. Finally, the new model is applied to the population forecast of Chongqing, China. The prediction results suggest that the population may reach a peak in 2020 and decline in the future. This finding is consistent with the logistic population growth model.
\end{abstract}

\section{Introduction}

Generally, a population that is too large or too small is not conducive to the overall development of a country. In 1979, British economist Thomas Robert Malthus proposed two well-known views of growth. Without hindrance, the population will grow geometrically, while the social resources on which humans depend for survival will grow arithmetically [1]. The Malthus population growth model is one of two classical population growth models; the population growth calculated by this model is infinite, but the balance between the population and social resources is disturbed after a period of unlimited population growth. Humans face various problems caused by the shortage of social resources. DeFries' et al. [2] studied the problem of station failures driven by urban population growth and agricultural trade in the first two centuries. Bloom and Freeman [3] studied the effects of rapid population growth on the labor supply and employment in developing countries. Abundant data indicate that a population that is too large will have many negative effects on development.
Furthermore, with a decrease in a population, the aging of the population is a significant problem. Currently, the aging problem generally occurs in developed countries and some developing countries. In the absence of response measures, there may be a shortage in the labor force and national defense forces. It is well known that China is a country with a large population, but the problem of aging in China's population is worsening; thus, the labor force is also shrinking. Zhong [4] studied the impact of population aging on income inequality in developing countries using the situation in rural China as an example, showing that the phenomenon of population aging exerts a serious impact on income inequality.

Therefore, the population not only affects economic development and environmental problems but also is closely related to social stability, labor employment, and the utilization of resources related to sustainable development. In light of the various influencing factors, it is particularly important to use scientific and accurate methods to predict and control the population and provide a reliable scientific basis for the government in making strategic population decisions [5]. 
In recent years, further progress in population prediction has been achieved; for example, Winiowski et al. [6] used the Bayesian extended Lee-Carter method to predict population growth. Raftery et al. [7] used Bayesian probability to predict the populations of all countries. Shang et al. [8] used the multifunctional level data method to predict the populations of all countries. The population of the United Kingdom has also been predicted. Bryant and Graham [9] used Bayesian demographic accounts to estimate the subnational population. Other papers have also contributed to population prediction $[10,11]$.

Currently, forecasting methods are generally divided into Bayesian methods [6], blocking growth models [12], and curve fitting [13]. Most methods are based on predicting the future population based on a time series. In addition to predicting population using a time series, the blocking growth model provides a particular description of the population's development. There are two main classical models: the Malthus population growth model and the logistic population growth model. Notably, the Malthus population growth model is suitable when the population is low and resources are abundant. In addition, in this model, the population grows infinitely. To solve this problem, Dutch biologist Verhulst introduced a constant, that is, $N_{m}$ (representing the maximum population that natural resources can accommodate), and proposed a logistic population growth model to avoid the problem of infinite population growth in the Malthus model. However, the logistic population growth model only needs three types of data, which can be used to obtain the two parameters of a binary nonlinear equation system. Because finding the analytical solution in a nonlinear equation system is very difficult and the results of the numerical solution may differ when using different methods, the prediction may be distorted. Therefore, this paper combines the differential equation of the logistic model with the modeling mechanism of the grey model equation and obtains the logistic grey prediction model.

Furthermore, the grey model is simple and adaptable and can better address mutation parameter changes because it does not need numerous data points to update predictions. Since its proposal, this prediction model [14] has been widely used in many fields, such as industry, agriculture, transportation, medicine, and military applications [15-24]. Researchers have conducted many in-depth and systematic studies based on the grey prediction model with respect to the initial values, background values, modeling mechanisms, model nature, and model combinations [25-28], which have promoted the development and perfection of its theoretical system. The grey model is also extended to the GM $(1, N)$, $\operatorname{DGM}(1,1)$, NDGM $(1,1)$, and CM $(1,1)$ power models and other new prediction model categories [29-33]. In addition, the ability of the grey forecasting model in population forecasting has also been studied [34].

In summary, starting with the classical logistic population growth model and considering the characteristics of the model and modeling mechanism of the grey prediction model, this paper establishes a grey prediction model based on the logistic population growth model which can predict the size of a population and estimate the maximum population capacity of a region through the least-squares method. Furthermore, to improve the accuracy of the logistic population growth grey prediction model (LPGM), a weak buffering operator is introduced, and a grey prediction model based on the weakened buffer operator's logistic population growth model is proposed. The empirical analysis shows that the established grey prediction model (LGM) calculates the population maximum capacity, that is, $N_{m}$, better than the logistic population model. The growth model is more realistic. The empirical analysis of the logistic population grey model with a weakening buffer operator (LPGMWBO) shows that its simulation effect is better than that of the LGM. The simulation accuracy is approximately $4.5 \%$. The simulation results show that the model can predict the expected population in the upcoming years, the trend of the prediction results, and the logistic population growth.

This paper makes the following main innovation points:

(1) In accordance with the differential equations of two classical models of population, the Malthus population growth model and the logistic population growth model, the grey prediction model is established through the grey difference information theory, that is, the relationship between the differential equation and the difference equation.

(2) The new model uses the modeling mechanism of the grey model, the least-squares method to estimate the maximum population capacity $N_{m}$, and the weakening buffer operator to optimize the accuracy of the new model. The population data of four regions in China are used to illustrate the effectiveness of the model. Finally, the paper demonstrates that the new model can effectively predict the population of Chongqing.

(3) On the basis of the Malthus model, the new model not only addresses the unrealistic assumption that the total population will continue to increase but also solves the problem of distortion encountered by the results of the logistic model, thereby allowing the new model to predict the population trend effectively.

In the following sections, we use symbols with various meanings, the corresponding definitions of which are listed in Table 1.

Similarly, the equations and their meanings are listed in Table 2.

The other sections in this paper are arranged as follows. In Section 2, the modeling mechanism of the grey prediction model is combined with the nature of the logistic population growth model to establish the LPGM. Then, the LPGM is joined with a weakening buffer operator to establish the LPGMWBO, following which the nature of the models is studied. Section 3 uses the population data of four provinces and cities in China to calculate six evaluation indicators to analyze the validity of the new model. Section 4 describes the empirical analysis using the LPGM to predict the maximum population maximum capacity, $N_{m}$, and the LPGMWBO to 
TABLE 1: Symbols and their meanings.

\begin{tabular}{lcc}
\hline & & Index symbol meanings \\
\hline 1 & $X^{(0)}$ & An original time sequence \\
2 & $X^{(1)}$ & 1-Accumulating generation operator (AGO) sequence of $X^{(0)}$ \\
3 & $Z^{(1)}$ & The mean sequence generated by consecutive neighbors of $X^{(1)}$ \\
5 & $Y^{(0)}$ & 1-Weakening buffer operator sequence of $X^{(0)}$ \\
6 & $Y^{(1)}$ & 1-Accumulating generation operator $(\mathrm{AGO})$ sequence of $Y^{(0)}$ \\
7 & $\bar{Z}^{(1)}$ & The mean sequence generated by consecutive neighbors of $Y^{(1)}$ \\
8 & $\widehat{Y}^{(0)}$ & The simulation time sequence of $Y^{(0)}$ \\
9 & $\varepsilon$ & The error sequence $Y^{(0)}$ \\
\hline
\end{tabular}

TABLE 2: Symbols and their definitions.

\begin{tabular}{lcc}
\hline & \multicolumn{2}{c}{ Index symbol definitions } \\
Index & $\mathrm{d} N / \mathrm{d} t=r N$ & Definition \\
\hline 1 & $\mathrm{~d} N / \mathrm{d} t=r N\left(1-N / N_{m}\right)$ & The Malthus population growth model \\
2 & $\mathrm{~d} N^{(1)}(t) / \mathrm{d} t+a N^{(1)}(t)=b\left[N^{(1)}(t)\right]^{2}$ & The logistic population growth model \\
3 & $N^{(0)}(k)+a z^{(1)}(k)=b\left[z^{(1)}(k)\right]^{2}$ & The logistic population grey model \\
4 & $Y^{(0)}(k)+\hat{a} \bar{z}^{(1)}(k)=\widehat{b}\left[\bar{z}^{(1)}(k)\right]^{2}$ & The grey Verhulst model \\
5 & & The logistic population grey model with a weakening buffer operator \\
\hline
\end{tabular}

simulate and predict the urban population in Chongqing, China. Section 5 presents the conclusion.

\section{Establishment of the Grey Prediction Model of Logistic Population Growth}

The Malthus model and the logistic model are classical models of population growth (see Appendix A for relevant information about the two models). The logistic growth model is as follows:

$$
\frac{\mathrm{d} N}{\mathrm{~d} t}=r N\left(1-\frac{N}{N_{m}}\right) .
$$

Equation (1) is a Bernoulli equation whose solution can be obtained as follows:

$$
N(t)=\frac{N_{m}}{1+\left(\left(N_{m} / N_{0}\right)-1\right) e^{-r\left(t-t_{0}\right)}},
$$

where the constant $N_{m}$ is the environmental capacity. In this section, the grey prediction model of logistic population growth is established according to the logistic model of population growth, and the corresponding optimization model is established according to the weakening buffer operator.

This section will establish the grey prediction model of logistic population growth based on the population growth logistic model and will establish a corresponding optimization model based on the weakening buffer operator.

2.1. Grey Prediction Model of Logistic Population Growth. According to equation (1), the following equation holds:

$$
\frac{\mathrm{d} N}{\mathrm{~d} t}-r N=-\frac{r}{N_{m}} N^{2} .
$$

Let $a=-r, b=-r / N_{m}$. Thus, we can observe the following:

$$
\frac{\mathrm{d} N}{\mathrm{~d} t}+a N=b N^{2}
$$

Set the number of people during the same time period as follows:

$$
N^{(0)}=\left\{N^{(0)}(1), N^{(0)}(2), \cdots, N^{(0)}(n)\right\} .
$$

The one-time cumulative sequence is as follows:

$$
N^{(1)}=\left\{N^{(1)}(1), N^{(1)}(2), \cdots, N^{(1)}(n)\right\},
$$

where $N^{(1)}(k)=\sum_{m=1}^{k} N^{(0)}(m)$. According to formula (4), the differential equation of the population increment $N(t)$ at time $t$ is as follows:

$$
\frac{\mathrm{d} N^{(1)}(t)}{\mathrm{d} t}+a N^{(1)}(t)=b\left[N^{(1)}(t)\right]^{2} .
$$

If the first-order difference is replaced by the left differential in (7), when $t=k$, we obtain the following:

$$
\begin{aligned}
\left.\frac{\mathrm{d} N^{(1)}(t)}{\mathrm{d} t}\right|_{t=k} & \left.\approx \frac{\Delta N^{(1)}(t)}{\Delta t}\right|_{t=k}=\frac{N^{(1)}(k)-N^{(1)}(k-1)}{k-(k-1)} \\
& =N^{(1)}(k)-N^{(1)}(k-1) \\
& =N^{(0)}(k) .
\end{aligned}
$$

Thus, the following grey model can be defined.

Definition 1. If sequences $N^{(0)}$ and $N^{(1)}$ are represented as formulas (5) and (6), respectively, sequence $Z^{(1)}$ is called the nearest mean generating sequence of $N^{(1)}$. 


$$
Z^{(1)}=\left\{z^{(1)}(1), z^{(1)}(2), \cdots, z^{(1)}(n)\right\},
$$

where $z^{(1)}(k)=\left(N^{(1)}(k)+N^{(1)}(k-1)\right) / 2$.

Definition 2. If sequences $N^{(0)}, N^{(1)}$, and $Z^{(1)}$ are as shown in (5), (6), and (9), respectively,

$$
N^{(0)}(k)+a z^{(1)}(k)=b\left[z^{(1)}(k)\right]^{2}
$$

is called the logistic grey model and is abbreviated as LGM $(1,1)$. Furthermore, the differential equation in formula (10) is called the whitening equation of the $\operatorname{LPGM}(1,1)$.

Formula (10) is consistent with the Verhulst model, and its left end is consistent with the whitening equation of the model in formula (4). This equation can be regarded as a whitening equation of a grey model. Furthermore, if the initial time point is $t=1$, the population between the time periods $[1, t]$ is expressed as follows:

$$
N^{(1)}(k)=\sum_{j=1}^{t} N^{(0)}(j) \text {. }
$$

It can be observed that the forms of formula (11) and the 1 -AGO sequence in formula (5) are exactly the same. Thus, if the discrete sequence $\left\{N^{(0)}(1), N^{(0)}(2), \cdots, N^{(0)}(k)\right\}$ is regarded as the original sequence, $\left\{N^{(1)}(1), N^{(1)}(2), \cdots\right.$, $\left.N^{(1)}(k)\right\}$ is its 1 -AGO sequence. Therefore, formula (10) is formally the same as the whitening equation of the Verhulst model and has the same connotation. The differential equation can thus be transformed into a grey model based on the principle of differential information to solve the population growth problem.

Definition 3. Let $Z^{(1)}=\left(z^{(1)}(2), z^{(1)}(3), \cdots, z^{(1)}(n)\right)$ be the mean generation sequence of accumulative sequence $N^{(1)}$ generated by population sequence $N^{(0)}$, and let

$$
\begin{aligned}
& B=\left[\begin{array}{cc}
-z^{(1)}(2) & \left(z^{(1)}(2)\right)^{2} \\
-z^{(1)}(3) & \left(z^{(1)}(3)\right)^{2} \\
\vdots & \vdots \\
-z^{(1)}(n) & \left(z^{(1)}(n)\right)^{2}
\end{array}\right], \\
& X=\left[\begin{array}{c}
N^{(0)}(2) \\
N^{(0)}(3) \\
\vdots \\
N^{(0)}(n)
\end{array}\right] \\
& P=\left[\begin{array}{l}
a \\
b
\end{array}\right] .
\end{aligned}
$$

Then, from formula (10), the least-squares estimate of parameters $a, b$ satisfies the following:

$$
\begin{aligned}
P & =[a, b]^{T} \\
& =\left(B^{T} B\right)^{-1} B^{T} X .
\end{aligned}
$$

The time response equation is as follows:

$$
\widehat{N}^{(1)}(k+1)=\frac{a x^{(1)}(1)}{b x^{(1)}(1)+\left(a-b x^{(1)}(1)\right) e^{a k}}
$$

The cumulative reduction value is as follows:

$$
\widehat{N}^{(0)}(k)=\widehat{N}^{(1)}(k)-\widehat{N}^{(1)}(k-1), \quad k=2,3, \cdots, n \text {. }
$$

Proof. By inputting data into formula (10), which is $N^{(0)}(k)+a z^{(1)}(k)=b\left[z^{(1)}(k)\right]^{2}$, we can obtain the following:

$$
\begin{gathered}
N^{(0)}(2)+a z^{(1)}(2)=b\left[z^{(1)}(2)\right]^{2}, \\
N^{(0)}(3)+a z^{(1)}(3)=b\left[z^{(1)}(3)\right]^{2}, \\
\vdots \\
N^{(0)}(n)+a z^{(1)}(n)=b\left[z^{(1)}(n)\right]^{2} .
\end{gathered}
$$

The matrix form of the above equation sets is as follows:

$$
Y=B P \text {. }
$$

For $a, b$, by substituting $N^{(0)}(k)$ with $-a z^{(1)}(k)^{2}+b\left[z^{(1)}(k)\right]^{2}, k=2,3, \cdots, n$, we can obtain the following error sequence:

$$
\varepsilon=Y-B P
$$

Let

$$
\begin{aligned}
s & =\mathcal{E}^{T} \mathcal{\varepsilon} \\
& =(Y-B P)^{T}(Y-B P) \\
& =\sum_{k=2}^{n}\left(-a z^{(1)}(k)+b\left[z^{(1)}(k)\right]^{2}-N^{(0)}(k)\right)^{2} \\
& =\sum_{k=2}^{n}\left(N^{(0)}(k)+a z^{(1)}(k)-b\left[z^{(1)}(k)\right]^{2}\right)^{2} .
\end{aligned}
$$

Then, the parameter list $\widehat{P}=[a, b]^{T}$ that minimizes $s$ should satisfy the following:

$$
\left\{\begin{array}{l}
\frac{\partial s}{\partial a}=2 \sum_{k=2}^{n}\left[N^{(0)}(k)+a z^{(1)}(k)-b\left[z^{(1)}(k)\right]^{2}\right] z^{(1)}(k)=0 \\
\frac{\partial s}{\partial b}=-2 \sum_{k=2}^{n}\left[N^{(0)}(k)+a z^{(1)}(k)-b\left[z^{(1)}(k)\right]^{2}\right]\left[z^{(1)}(k)\right]^{2}=0 .
\end{array}\right.
$$

Thus,

$$
\left\{\begin{array}{l}
\sum_{k=2}^{n}\left[N^{(0)}(k)+a z^{(1)}(k)-b\left[z^{(1)}(k)\right]^{2}\right] z^{(1)}(k)=0, \\
\sum_{k=2}^{n}\left[N^{(0)}(k)+a z^{(1)}(k)-b\left[z^{(1)}(k)\right]^{2}\right]\left[z^{(1)}(k)\right]^{2}=0 .
\end{array}\right.
$$

Therefore, we obtain the following: 


$$
\left\{\begin{array}{l}
a=\frac{\sum_{k=2}^{n}\left[z^{(1)}(k)\right]^{4} \sum_{k=2}^{n} N^{(0)}(k) z^{(1)}(k)-\left(\sum_{k=2}^{n}\left[z^{(1)}(k)\right]^{3}\right)^{2} \sum_{k=2}^{n} N^{(0)}(k)\left[z^{(1)}(k)\right]^{2}}{\sum_{k=2}^{n}\left[z^{(1)}(k)\right]^{3}\left(\sum_{k=2}^{n}\left[z^{(1)}(k)\right]^{3}\right)^{2}-\sum_{k=2}^{n}\left[z^{(1)}(k)\right]^{4} \sum_{k=2}^{n} z^{(1)}(k)}, \\
b=\frac{\sum_{k=2}^{n}\left[z^{(1)}(k)\right]^{3} \sum_{k=2}^{n} N^{(0)}(k) z^{(1)}(k)-\sum_{k=2}^{n}\left[z^{(1)}(k)\right] \sum_{k=2}^{n} N^{(0)}(k)\left[z^{(1)}(k)\right]^{2}}{\left(\sum_{k=2}^{n}\left[z^{(1)}(k)\right]^{3}\right)^{2}-\sum_{k=2}^{n}\left[z^{(1)}(k)\right]^{4} \sum_{k=2}^{n} z^{(1)}(k)}
\end{array}\right.
$$

Thus,

$$
\begin{aligned}
P & =[a, b]^{T} \\
& =\left(B^{T} B\right)^{-1} B^{T} Y .
\end{aligned}
$$

The following is true for the maximum capacity $N_{m}$.

Definition 4. Maximum capacity $N_{m}=a / b$.

Proof. From formula (2), we can determine that $a=-r, b=-r / N_{m}$, and $N_{m}=a / b$ can be obtained.

2.2. LPGM Based on a Weakening Buffer Operator. The LPGM is a classical grey Verhulst model. There may be disturbances in the original population data that may lead to inconsistencies between the quantitative prediction results and the intuitive qualitative analysis conclusions. Therefore, the data should first be preprocessed. Because the LPGM is a classical grey Verhulst model, which is a grey power model, the buffer operator is weakened by the following power exponents.

Definition 5 (see [16]). Let $X=(x(1), x(2), \cdots, x(n))$ be a nonnegative sequence of the system behavior data, and let $X D=(x(1) d, x(2) d, \cdots, x(n) d)$. Here,

$$
\begin{aligned}
x(k) d & =\left(\frac{(x(k))^{\beta}+(x(k+1))^{\beta}+\cdots+(x(n))^{\beta}}{n-k+1}\right)^{1 / \beta} \\
& =\left(\frac{1}{n-k+1} \sum_{i=k}^{n}(x(i))^{\beta}\right)^{1 / \beta}
\end{aligned}
$$

where $k=1,2, \cdots, n, \beta \neq 0$. Then, when $X$ is a monotonic growth sequence, a monotonic attenuation sequence or a concussion sequence, that is, $D$, is a weakening buffer operator; $D$ is called the power average weakening buffer operator and is denoted as PAWBO.

Parameter $\beta$ has the function of adjusting the effect of the weakening buffer operator. In a monotonic growth sequence, the effect of PAWBO varies in the same direction as $\beta$. If PAWBO is slowly weakened, a smaller $\beta$ can be used. In a monotonic attenuation sequence, the action of the weakening operator shrinks the data, and the attenuation of the weakening buffer sequence is slower than that of the original data sequence. The effect of PAWBO on monotonic attenuation sequences varies in the opposite direction of $\beta$. If PAWBO is slowly weakened, a larger $\beta$ can be used.

The LPGMWBO using the LPGM sequence buffer operator is as follows. Set the population sizes during equal periods as follows:

$$
N^{(0)}=\left\{N^{(0)}(1), N^{(0)}(2), \cdots, N^{(0)}(n)\right\} .
$$

Set the sequences with the effect of the buffer operator PAWBO as follows:

$$
N^{(0)}(D)=Y^{(0)}=\left\{N^{(0)}(1) d, N^{(0)}(2) d, \cdots, N^{(0)}(n) d\right\},
$$

and here, $N^{(0)}(k) d=\left(\left(\left(N^{(0)}(k)\right)^{\beta}+\left(N^{(0)}(k+1)\right)^{\beta}+\cdots+\right.\right.$ $\left.\left.\left(N^{(0)}(n)\right)^{\beta}\right) /(n-k+1)\right)^{1 / \beta}$.

Therefore,

$$
Y^{(0)}=\left(\begin{array}{c}
{\left[y^{(0)}(1)\right]^{\beta}} \\
{\left[y^{(0)}(2)\right]^{\beta}} \\
\vdots \\
{\left[y^{(0)}(n)\right]^{\beta}}
\end{array}\right)\left(\begin{array}{ccccc}
\frac{1}{n} & \frac{1}{n} & \frac{1}{n} & \cdots & \frac{1}{n} \\
0 & \frac{1}{n-1} \frac{1}{n-1} & \cdots & \frac{1}{n-1} \\
\vdots & \vdots & \vdots & \vdots & \vdots \\
0 & 0 & \frac{1}{n-2} & \cdots & \frac{1}{n-2} \\
0 & 0 & 0 & 0 & 1
\end{array}\right)\left(\begin{array}{c}
{\left[N^{(0)}(1)\right]^{\beta}} \\
{\left[N^{(0)}(2)\right]^{\beta}} \\
\vdots \\
{\left[N^{(0)}(n)\right]^{\beta}}
\end{array}\right)
$$$$
=Y A N \text {, }
$$

and the cumulative sequence of $Y^{(0)}$ is as follows:

$$
Y^{(1)}=\left\{Y^{(1)}(1), Y^{(1)}(2), \cdots, Y^{(1)}(n)\right\}
$$

where $Y^{(1)}(k)=\sum_{m=1}^{k} Y^{(0)}(m)$, generating the sequence of the means.

$$
\bar{Z}^{(1)}=\left\{\bar{z}^{(1)}(1), \bar{z}^{(1)}(2), \cdots, \bar{z}^{(1)}(n)\right\},
$$

where $\bar{z}^{(1)}(k)=\left(Y^{(1)}(k)+Y^{(1)}(k-1)\right) / 2$.

Then, the LGM under the buffer operator is as follows:

$$
Y^{(0)}(k)+\widehat{a} \bar{z}^{(1)}(k)=\widehat{b}\left[\bar{z}^{(1)}(k)\right]^{2} .
$$

Due to Definition 3.1, we let 


$$
\begin{aligned}
C & =\left[\begin{array}{cc}
-\bar{z}^{(1)}(2) & \left(\bar{z}^{(1)}(2)\right)^{2} \\
-\bar{z}^{(1)}(3) & \left(\bar{z}^{(1)}(3)\right)^{2} \\
\vdots & \vdots \\
-\bar{z}^{(1)}(n) & \left(\bar{z}^{(1)}(n)\right)^{2}
\end{array}\right], \\
\bar{Y} & =\left[\begin{array}{c}
y^{(0)}(2) \\
y^{(0)}(3) \\
\vdots \\
y^{(0)}(n)
\end{array}\right], \\
\widehat{P} & =\left[\begin{array}{c}
\widehat{a} \\
\widehat{b}
\end{array}\right] .
\end{aligned}
$$

Then, the least-squares estimation of the parameters in (3.8) $a, b$ satisfies the following:

$$
\begin{aligned}
\widehat{P} & =[\widehat{a}, \widehat{b}]^{T} \\
& =\left(C^{T} C\right)^{-1} C^{T} \bar{Y} .
\end{aligned}
$$

The time response equation is as follows:

$$
\widehat{y}^{(1)}(k+1)=\frac{a y^{(1)}(1)}{b y^{(1)}(1)+\left(a-b y^{(1)}(1)\right) e^{\mathrm{ak}}} .
$$

The cumulative reduction value is as follows:

$$
\hat{y}^{(0)}(k)=\hat{y}^{(1)}(k)-\hat{y}^{(1)}(k-1), \quad k=2,3, \cdots, n .
$$

Through defining the LPGMWBO and using Theorems 3.1 and 3.3, the flowchart of the LPGMWBO can be obtained, as shown in Figure 1.

\section{Validation of the LPGMWBO}

3.1. Numerical Simulation Experiments. To illustrate the effectiveness of the model, this section uses the population data of four provinces in China as reference data. The data come from http://data.stats.gov.cn/easy query.htm? $\mathrm{Cn}=\mathrm{E} 0103$, including the total population of the four regions of Beijing, Shanghai, Guangdong, and Sichuan. The comparison models are the classic Verhulst model and the NGM $(1,1)$ model. In addition to the MAPE value commonly used in the grey model, evaluation indexes such as RMSPE, IA, U1, $\mathrm{U} 2$, and $R$ are also introduced. The specific meanings and calculation formulas are shown in Table 3. The smaller the values of MAPE, RMSPE, U1, and U2 are, the higher the accuracy of the model is, and vice versa. The values of IA and $R$ are the opposite, such that the higher the value is, the higher the accuracy of the model is. The population data for Beijing and Shanghai are selected for the comparison of the fitting effect. The data are given in Table 4 . The population data of Guangdong and Sichuan province are compared with the prediction effect. These data are given in Table 5. An effect diagram is used to compare the effects of each model.

The LPGMWBO uses the first row of data in Table 4 to perform calculations through MATLAB in accordance with

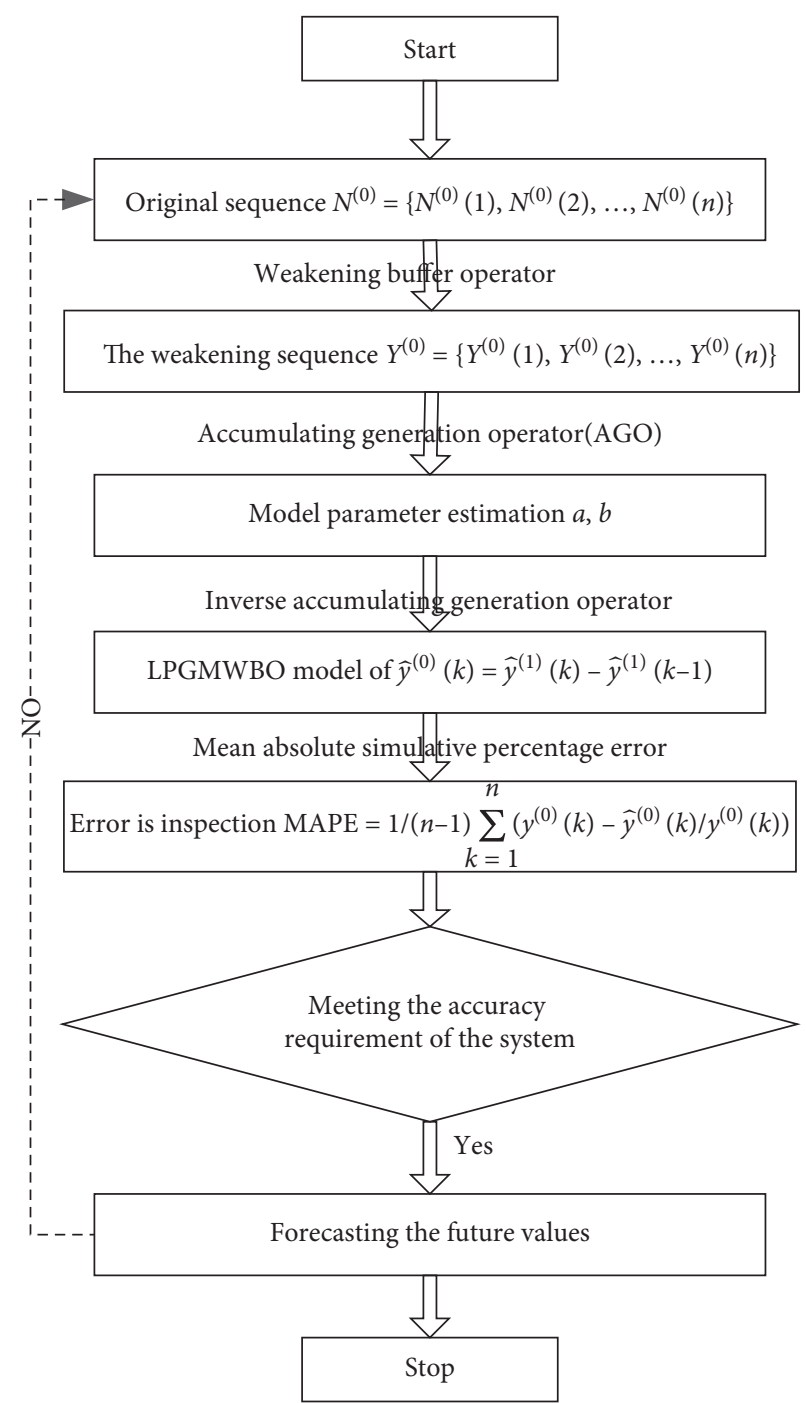

FIGURE 1: Flowchart of the LPGMWBO.

the steps in the forecast flowchart. The results are calculated to four decimal places. The calculation of other models is also completed through this software. The specific steps are as follows:

(i) Data processing

$$
\begin{aligned}
N^{(0)} & =\left(N^{(0)}(1), N^{(0)}(2), \cdots, N^{(0)}(5)\right) \\
& =(1825,1858,1877,1880,1878) .
\end{aligned}
$$

According to Definition 3,

$$
\begin{aligned}
N^{(0)}(D)= & Y^{(0)} \\
= & \left\{y^{(0)}(1), y^{(0)}(2), \cdots, y^{(0)}(5)\right\} \\
= & \{16560.524,12296.758,8344.233, \\
& 4814.226,1878\},
\end{aligned}
$$

where $\beta=0.42$. The $1-\mathrm{AGO}$ sequences $Y^{(1)}(k)=$ $\sum_{m=1}^{k} Y^{(0)}(m)$, 
TABle 3: Metrics for evaluating effectiveness of the models [34].

\begin{tabular}{|c|c|c|}
\hline Name & Abbreviation & Formulation \\
\hline Absolute percentage error & APE & $X^{(0)}(k)-\widehat{X}^{(0)}(k) / X^{(0)}(k) \times 100 \%$ \\
\hline Mean absolute percentage error & MAPE & $1 /(n-1) \sum_{k=1}^{n}\left|\left(X^{(0)}(k)-\widehat{X}^{(0)}(k)\right) / X^{(0)}(k)\right| \times 100 \%$ \\
\hline Root mean squares percentage error & RMSPE & $\sqrt{1 / n \sum_{i=1}^{n}\left(\left(X^{(0)}(k)-\widehat{X}^{(0)}(k)\right) / X^{(0)}(k)\right)^{2}} \times 100 \%$ \\
\hline Index of agreement & IA & $1-\left(\sum_{k=1}^{n}\left(X^{(0)}(k)-\widehat{X}^{(0)}(k)\right)^{2}\right) /\left(\sum_{k=1}^{n}\left(\left|\widehat{X}^{(0)}(k)-\bar{x}\right|+\left|X^{(0)}(k)-\bar{x}\right|\right)^{2}\right)$ \\
\hline Theil U statistic 1 & U1 & $\left(\sqrt{1 / n \sum_{i=1}^{n}\left(X^{(0)}(k)-\widehat{X}^{(0)}(k)\right)^{2}}\right) / \sqrt{1 / n \sum_{i=1}^{n}\left[X^{(0)}(k)\right]^{2}}+\sqrt{1 / n \sum_{i=1}^{n}\left[\widehat{X}^{(0)}(k)\right]^{2}}$ \\
\hline Theil U statistic 2 & $\mathrm{U} 2$ & $\left(\left[\sum_{i=1}^{n}\left(X^{(0)}(k)-\widehat{X}^{(0)}(k)\right)^{2}\right]^{1 / 2}\right) /\left(\left[\sum_{i=1}^{n}\left[X^{(0)}(k)\right]^{2}\right]^{1 / 2}\right)$ \\
\hline Correlation coefficient & $R$ & $\operatorname{Cov}\left(\hat{X}^{(0)}, X^{(0)}\right) / \sqrt{\operatorname{Var}\left(\hat{X}^{(0)}\right) \operatorname{Var}\left(X^{(0)}\right)}$ \\
\hline
\end{tabular}

TAble 4: Population of Beijing and Shanghai from 2013 to 2017 (unit: ten thousand).

\begin{tabular}{llllll}
\hline Year & 2013 & 2014 & 2015 & 2016 & 2017 \\
\hline Beijing & 1825 & 1858 & 1877 & 1880 & 1878 \\
Shanghai & 2164 & 2173 & 2116 & 2127 & 2121 \\
\hline
\end{tabular}

Table 5: Population of Guangdong and Sichuan from 2013 to 2017 (unit: ten thousand).

\begin{tabular}{lllllll}
\hline Year & 2012 & 2013 & 2014 & 2015 & 2016 & 2017 \\
\hline Guangdong & 7140 & 7212 & 7292 & 7454 & 7611 & 7802 \\
Sichuan & 3516 & 3640 & 3769 & 3912 & 4066 & 4217 \\
\hline
\end{tabular}

$$
\begin{aligned}
Y^{(1)}= & \left\{y^{(1)}(1), y^{(1)}(2), \cdots, y^{(1)}(5)\right\} \\
= & \{16560.524,28857.282,37201.516, \\
& 42015.732,43893.732\} .
\end{aligned}
$$

The mean sequence generated by consecutive neighbors $Z^{(1)}$ of $N^{(1)}$ is given by

$$
\begin{aligned}
Z^{(1)}= & \left\{z^{(1)}(2), z^{(1)}(3), \cdots, y^{(1)}(5)\right\} \\
= & \{22708.903,33029.399,39608.624, \\
& 42954.732\} .
\end{aligned}
$$

(ii) Parameter estimation

According to Theorem 3.1, the matrices $B$ and $Y$ are given by

$$
\begin{aligned}
& C=\left[\begin{array}{cc}
-\bar{z}^{(1)}(2) & \left(\bar{z}^{(1)}(2)\right)^{2} \\
-\bar{z}^{(1)}(3) & \left(\bar{z}^{(1)}(3)\right)^{2} \\
\vdots & \vdots \\
-\bar{z}^{(1)}(n) & \left(\bar{z}^{(1)}(n)\right)^{2}
\end{array}\right], \\
& \bar{Y}=\left[\begin{array}{c}
y^{(0)}(2) \\
y^{(0)}(3) \\
\vdots \\
y^{(0)}(n)
\end{array}\right], \\
& \widehat{P}=\left[\begin{array}{c}
\widehat{a} \\
\widehat{b}
\end{array}\right] .
\end{aligned}
$$

The parameters list $\widehat{P}=[\hat{a}, \widehat{b}]^{T}$ is given by

$$
\begin{aligned}
\widehat{P} & =[\widehat{a}, \widehat{b}]^{T} \\
& =\left(C^{T} C\right)^{-1} C^{T} \bar{Y} \\
& =\left[-1.05926,-2.37492 \times 10^{-5}\right] .
\end{aligned}
$$

(iii) Calculate the simulation value.

By substituting parameter values according to Step 2,

$$
\hat{y}^{(1)}(k+1)=\frac{a y^{(1)}(1)}{b y^{(1)}(1)+\left(a-b y^{(1)}(1)\right) e^{\mathrm{ak}}} .
$$

(iv) Calculate the simulation values and errors. The results are given in Table 6.

Table 6 shows that the six evaluation indicators of the LPGMWBO all offer the best results. To show the differences of various models more intuitively, the trend graph and effect comparison graph of the model simulation results in Table 6 and the original data are given in Figure 2. The APE values of the four points in the LPGMWBO are all low in Figure 2, so MAPE is the lowest.

The comparison models are the Verhulst model and the NGM $(1,1)$ model. The evaluation indexes are still MAPE, RMSPE, IA, and U1, the Shanghai data from 2013 to 2017 are used as fitting data, the population data of Guangzhou and Sichuan from 2012 to 2016 are used as fitting data, and the data from 2017 are used as forecast data. The specific results are given in Tables 7-9.

The six comparison indicators of the fitting effect in Table 7 show that the LPGMWBO is the best among the three models; Table 8 shows that the MAPE values of the LPGMWBO are the lowest, and the other five indicators of the LPGMWBO are also the best. In Table 9, the best fittingeffect model is the NGM $(1,1)$, and the best prediction-effect model is the LPGMWBO. The results for the other five comparison indexes of the LPGMWBO are slightly worse than those of the NGM $(1,1)$, but the effect of the LPGMWBO is good. According to Tables 7-9, error comparison charts are drawn, as shown in Figures 3-5. The LPGMWBO in Figure 3 has a one-year APE that is slightly larger, while the other three years are the smallest. Figure 4 shows that the APE values of the LPGMWBO in 2014 and 
TABLE 6: Fitting metrics of models for validation of Beijing data.

\begin{tabular}{|c|c|c|c|c|c|c|c|}
\hline Year & Raw data & $\operatorname{NGM}(1,1)$ & APE (\%) & Verhulst & APE (\%) & LPGMWBO & APE (\%) \\
\hline 2013 & 1825 & 1825.00 & 0.0000 & 1825.00 & 0.0000 & 16560.52 & 0.0000 \\
\hline 2014 & 1858 & 1089.93 & 41.3386 & 1400.44 & 24.6266 & 11542.75 & -6.13179 \\
\hline 2015 & 1877 & 1736.99 & -7.4591 & 1918.76 & 2.2251 & 8955.60 & 7.326847 \\
\hline 2016 & 1880 & 1853.46 & -1.4117 & 2092.57 & 11.3071 & 4603.04 & -4.38653 \\
\hline 2017 & 1878 & 1874.42 & -0.1905 & 1783.56 & -5.0287 & 1874.89 & -0.16543 \\
\hline MAPE & & & 12.6000 & & 10.7971 & & 4.5026 \\
\hline RMSPE & & & 18.7965 & & 12.3657 & & 4.7021 \\
\hline IA & & & -0.2883 & & 0.0582 & & 0.9982 \\
\hline U1 & & & 0.0980 & & 0.0625 & & 0.0218 \\
\hline U2 & & & 0.1875 & & 0.1236 & & 0.0435 \\
\hline$R$ & & & 0.1329 & & 0.3120 & & 0.9966 \\
\hline
\end{tabular}

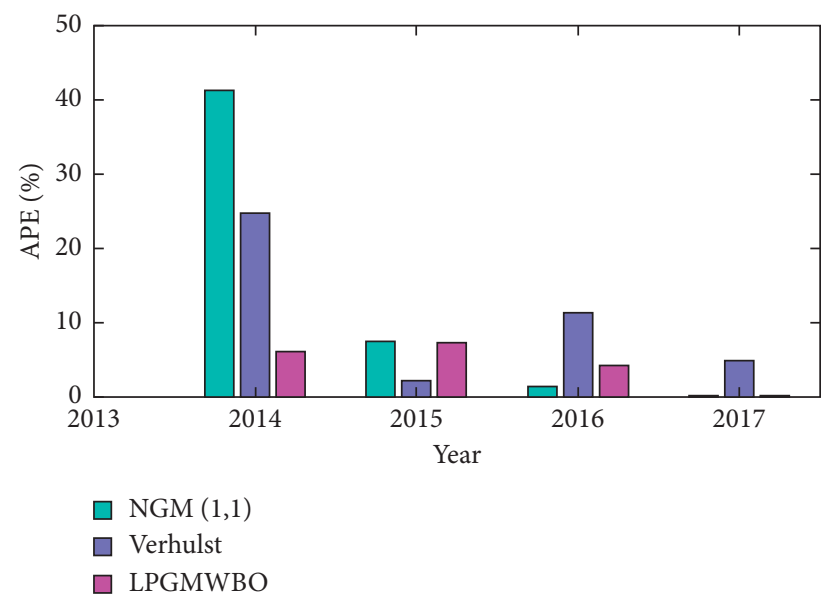

Figure 2: APE values of the three model validations of Beijing data.

Table 7: Fitting metrics of models for validation of Shanghai data.

\begin{tabular}{|c|c|c|c|c|c|c|c|}
\hline Year & Raw data & NGM & APE (\%) & Verhulst & APE (\%) & LPGMWBO & APE (\%) \\
\hline 2013 & 2164 & 2164.00 & 0.0000 & 2164.00 & 0.0000 & 19506.81 & 0.0000 \\
\hline 2014 & 2173 & 1174.22 & -45.9630 & 1626.21 & -25.1628 & 13445.81 & -6.0980 \\
\hline 2015 & 2116 & 2059.15 & -2.6868 & 2199.68 & 3.9544 & 10307.35 & 7.5003 \\
\hline 2016 & 2127 & 2119.36 & -0.3592 & 2371.28 & 11.4846 & 5244.43 & -4.6757 \\
\hline 2017 & 2121 & 2123.46 & 0.1158 & 2005.50 & -5.4454 & 2121.51 & 0.02410 \\
\hline MAPE & & & 12.2812 & & 11.5118 & & 4.5745 \\
\hline RMSPE & & & 20.5911 & & 12.7307 & & 4.8021 \\
\hline IA & & & -0.2538 & & -0.0230 & & 0.9982 \\
\hline U1 & & & 0.1090 & & 0.0651 & & 0.0218 \\
\hline $\mathrm{U} 2$ & & & 0.2090 & & 0.1286 & & 0.0435 \\
\hline$R$ & & & -0.6412 & & -0.6135 & & 0.9966 \\
\hline
\end{tabular}

2016 were slightly higher, while the APE values in other years were relatively small. Figure 5 shows that the LPGMWBO has higher APE values in 2013, 2014, and 2016, resulting in the simulated MAPE value not being the lowest, but the predicted value in 2017 is close to the true value, indicating that the new model is also effective.

3.2. Analysis of Results. MATLAB software is used to calculate the entire simulation process for four actual cases, combined with the six evaluation index results of the three models. In the first and second cases, the fitting effect of the LPGMWBO is the best of the three grey prediction models. The value of the fitting MAPE is lower than $5 \%$, which is an effective fitting. In Case 3, the fitting and prediction effect of the LPGMWBO is the best among the three models, and the six evaluation indicators are also the best. In Case 4, although the results of several indicators of the LPGMWBO are slightly worse than those of $\operatorname{NGM}(1,1)$, they do not affect the effectiveness of the model. Therefore, the LPGMWBO is an effective grey prediction model that can be applied to population forecasting. 
TABLE 8: Fitting metrics of models for validation of Guangdong data.

\begin{tabular}{|c|c|c|c|c|c|c|c|}
\hline Year & Raw data & NGM & APE (\%) & Verhulst & APE (\%) & LPGMWBO & APE (\%) \\
\hline 2012 & 7140 & 7140.00 & 0.0000 & 7140.00 & 0.0000 & 106568.00 & 0.0000 \\
\hline 2013 & 7212 & 8245.93 & 14.3362 & 5390.04 & -25.2628 & 74313.97 & -9.2282 \\
\hline 2014 & 7292 & 8662.91 & 18.8001 & 7435.44 & 1.9671 & 65607.91 & 10.7046 \\
\hline 2015 & 7454 & 9206.39 & 23.5095 & 8263.98 & 10.8664 & 39608.65 & 1.4071 \\
\hline 2016 & 7611 & 9914.81 & 30.2693 & 7243.32 & -4.8309 & 18694.31 & -13.4804 \\
\hline MAPE & & & 21.7287 & & 10.7318 & & 8.7051 \\
\hline 2017 & 7802 & 10838.16 & 38.9151 & 5140.91 & -34.1078 & 7796.16 & -0.0748 \\
\hline MAPE & & & 38.9151 & & 34.1078 & & 0.7484 \\
\hline RMSPE & & & 24.2977 & & 18.013 & & 7.9943 \\
\hline IA & & & -0.7220 & & -0.0784 & & 0.9962 \\
\hline $\mathrm{U} 1$ & & & 0.11214 & & 0.0957 & & 0.0337 \\
\hline $\mathrm{U} 2$ & & & 0.2493 & & 0.1842 & & 0.0670 \\
\hline$R$ & & & 0.9763 & & -0.2451 & & 0.9925 \\
\hline
\end{tabular}

TABLE 9: Fitting metrics of models for validation of Sichuan data.

\begin{tabular}{|c|c|c|c|c|c|c|c|}
\hline Year & Raw data & NGM & APE (\%) & Verhulst & APE (\%) & LPGMWBO & APE (\%) \\
\hline 2012 & 3516 & 3516.00 & 0.0000 & 3516.00 & 0.0000 & 57200.41 & 0.0000 \\
\hline 2013 & 3640 & 3740.85 & 2.7706 & 2694.63 & -25.9717 & 40138.75 & -9.2721 \\
\hline 2014 & 3769 & 3879.89 & 2.9421 & 3788.95 & 0.5293 & 35605.46 & 10.6183 \\
\hline 2015 & 3912 & 4031.68 & 3.0592 & 4318.81 & 10.3991 & 21571.71 & 1.4600 \\
\hline 2016 & 4066 & 4197.39 & 3.2313 & 3887.62 & -4.3871 & 10200.79 & -13.2837 \\
\hline MAPE & & & 3.0007 & & 10.3217 & & 8.6585 \\
\hline 2017 & 4217 & 4378.29 & 3.8248 & 2822.88 & -33.0595 & 4257.574 & 0.9621 \\
\hline MAPE & & & 3.8248 & & 33.0595 & & 0.9621 \\
\hline RMSPE & & & 2.908642 & & 17.77229 & & 7.939729 \\
\hline IA & & & 0.951637 & & 0.136366 & & 0.996091 \\
\hline U1 & & & 0.014756 & & 0.09594 & & 0.033772 \\
\hline $\mathrm{U} 2$ & & & 0.02992 & & 0.184214 & & 0.06721 \\
\hline$R$ & & & 0.996085 & & 0.031431 & & 0.992387 \\
\hline
\end{tabular}

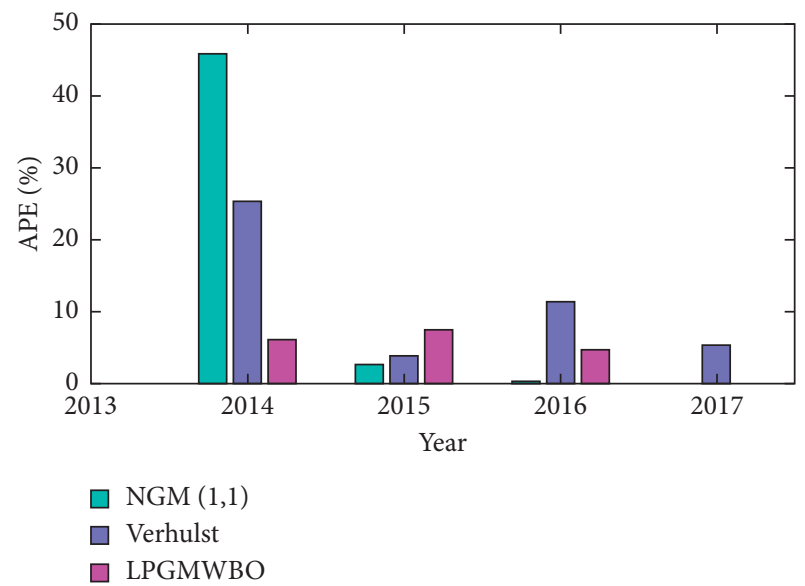

FIgURE 3: The APE of the three models for validation of Shanghai data.

\section{Applications}

4.1. Data Description. The website http://data.stats.gov.cn/ easyquery.htm? $\mathrm{Cn}=\mathrm{E} 0103$, which was used in this study, provides the population data for Chongqing's urban population from 2013 to 2017, as shown in Table 10.

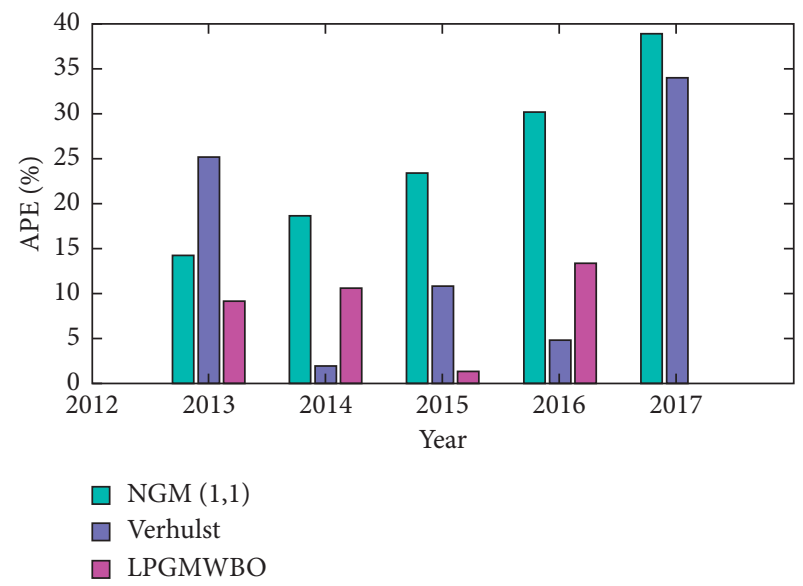

FIgURE 4: The APE of the three models for validation of Guangzhou data.

4.2. Data Description. First, according to the data shown in Table 10, the parameters of the model are estimated through the LPGM and the least-squares method. Then, we calculate the maximum population size $N_{m}$, which is compared to the maximum population size $N_{m}$ as calculated by the logistic population growth model, and the corresponding results are obtained. Second, the LPGM and LPGMWBO are calculated 


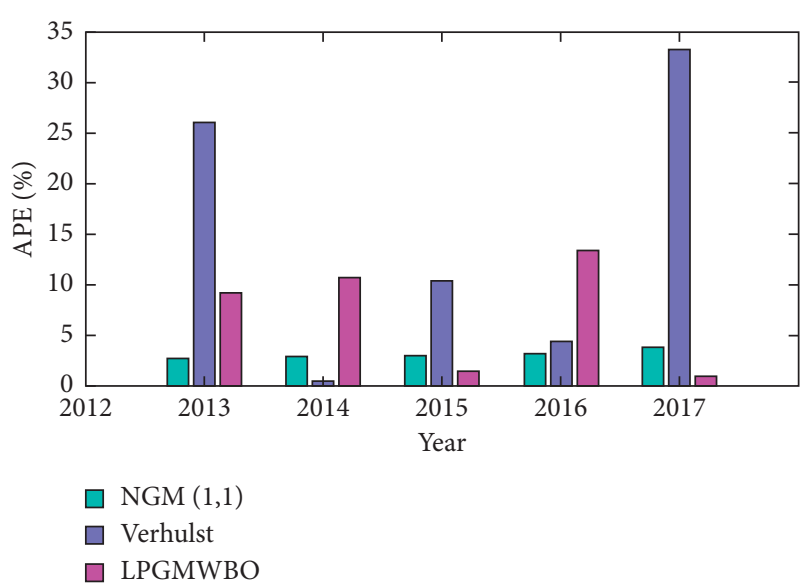

Figure 5: The APE of the three models for validation of Sichuan data.

TABle 10: Urban population in Chongqing, China, 2013-2017 (unit: ten thousand).

\begin{tabular}{llllll}
\hline Year & 2013 & 2014 & 2015 & 2016 & 2017 \\
\hline Population of Chongqing & 1733 & 1783 & 1838 & 1908 & 1971 \\
\hline
\end{tabular}

by using the data shown in Table 1 . Then, the results are compared.

According to the logistic population growth model (4) and the population data from 2013, 2014, and 2015, the following equations can be obtained:

$$
\left\{\begin{array}{l}
1783=\frac{N_{m}}{1+\left(\left(N_{m} / 1733\right)-1\right) e^{-r}}, \\
1838=\frac{N_{m}}{1+\left(\left(N_{m} / 1733\right)-1\right) e^{-2 r}} .
\end{array}\right.
$$

The maximum capacity $N_{m}$ of the numerical solution of the nonlinear equation can be calculated using MATLAB as follows: $N_{m}=987.6634$.

We use population data from 2013, 2015, and 2016 to calculate the population capacity $N_{m}$ as follows: $N_{m}=1451.4002$. Similarly, the populations in 2013, 2016, and 2017 can be used to determine that the numerical solution is not a real root.

According to the maximum capacity $N_{m}$ in the logistic population growth model, the calculated maximum capacities in the three cases are not consistent with the actual situation.

Subsequently, $a, b$ is calculated according to the leastsquares estimation of the LPGM; then, the maximum population $N_{m}$ is calculated, and the parameter list $P=$ $[a, b]^{T}$ is as follows:

$$
\begin{aligned}
P & =[a, b]^{T} \\
& =\left(B^{T} B\right)^{-1} B^{T} X \\
& =\left[-0.70462,-5.838 \times 10^{-5}\right] .
\end{aligned}
$$

Therefore, we obtain the following:

$$
\begin{aligned}
& a=-0.70462, \\
& b=-5.838 \times 10^{-5} .
\end{aligned}
$$

Definition 1 leads to a maximum capacity of $N_{m}=b / a=12068.7070$, and the results agree with the actual situation. The same data are used to calculate the maximum capacity $N_{m}$ of the logistic population growth model and the LPGM, which shows that the LPGM has better adaptability.

The following is an empirical analysis of the applicability of the LPGMWBO based on the data shown in Table 10. The calculation is based on the specific steps of the LPGMWBO shown in Figure 1. To illustrate the validity of the LPGMWBO, the MAPE values of the model are obtained according to the above steps and then compared with the experimental results of the LPGM, as shown in Table 11.

As shown in Table 11, the effect of buffering the original data is better than that of the original data. Thus, the effect of the LPGMWBO is substantially better than that of the LPGMWBO.

Using the data shown in Table 10, we illustrate the simulation results of the above two models in Figures 6(a) and $6(\mathrm{~b})$.

As shown in Figure 6, the simulation effect of the LPGMWBO is better than that of the LPGM.

As shown in Table 11, the MAPE of the LPGM is approximately $4.5 \%$. The grey model [29] is derived from the reference table. The LPGMWBO simulates the size of the urban population in Chongqing, and the accuracy in China is approximately $12 \%$. Therefore, the population can be predicted using the LPGMWBO. The prediction results are shown in Table 12.

Table 12 shows that the projected population in all years does not exceed the maximum capacity, but the maximum population may be reached by 2020 . When the maximum capacity is reached, the populations in 2021 and 2022 will decline, which is also consistent with the parameters of the logistic population growth model. More importantly, in the logistic population growth model (2), when $0<N<N_{m}$, we can obtain the following by deriving $t$ through the two extremes of formula (1):

$$
\frac{\mathrm{d}^{2} N}{\mathrm{~d} t^{2}}=r\left(1-\frac{2 N}{N_{m}}\right) \cdot \frac{\mathrm{d} N}{\mathrm{~d} t}
$$

As shown in formula (42), when $N<N_{m} / 2$ and $\mathrm{d}^{2} N / \mathrm{d} t^{2}>0$, it can be determined that $\mathrm{d} N / \mathrm{d} t$ is an additive function. When $N>N_{m} / 2$ and $\mathrm{d}^{2} N / \mathrm{d} t^{2}<0$, it can be determined that $\mathrm{dN} / \mathrm{d} t$ is a subtractive function. According to the extreme value discriminant, $\mathrm{d} N / \mathrm{d} t$ reaches the maximum value when $N=N_{m} / 2$. Therefore, when the population reaches $N=N_{m} / 2$, the population growth rate is the fastest. To further illustrate the growth rate observed in Table 12, we provide Figure 7.

Based on the population trend chart shown in Figure 7, when the population reaches 7195.16 in 2019, the growth rate is observed to be the fastest from 2018 to 2020, which is consistent with the growth trend of the logistic population 
TABLE 11: Simulated and MAPE values for the two models of urban population size in Chongqing, China.

\begin{tabular}{|c|c|c|c|c|c|}
\hline \multirow{2}{*}{$\begin{array}{l}\text { Actual value } \\
N^{(0)}(k)\end{array}$} & \multicolumn{2}{|c|}{$\begin{array}{l}\text { Logistic population grey } \\
\text { model (LPGM) }\end{array}$} & \multirow{2}{*}{$\begin{array}{l}\text { Weakening buffer } \\
\text { operator } Y^{(0)}(k)\end{array}$} & \multicolumn{2}{|c|}{$\begin{array}{l}\text { Logistic population grey model with a } \\
\text { weakening buffer operator (LPGMWBO) }\end{array}$} \\
\hline & $\begin{array}{c}\text { Simulation } \\
\text { value } N^{(0)}(k)\end{array}$ & $\begin{array}{l}\text { Simulation } \\
\text { error } \Delta_{k}(\%)\end{array}$ & & $\begin{array}{l}\text { Simulation value } \\
\qquad Y^{(0)}(k)\end{array}$ & $\begin{array}{c}\text { Simulation error } \\
\Delta_{k}(\%)\end{array}$ \\
\hline 1733 & 1733 & 0.0000 & 17035.30 & 17035.30 & 0.0000 \\
\hline 1783 & 1323 & 0.25749 & 12712.68 & 11929.15 & 0.06163 \\
\hline 1838 & 1854 & 0.00907 & 8686.13 & 9322.28 & 0.07323 \\
\hline 1908 & 2103 & 0.10269 & 5050.85 & 4824.62 & 0.04479 \\
\hline 1971 & 1884 & 0.04390 & 1971 & 1975.51 & 0.00229 \\
\hline & & 10.329 & & & 4.549 \\
\hline
\end{tabular}

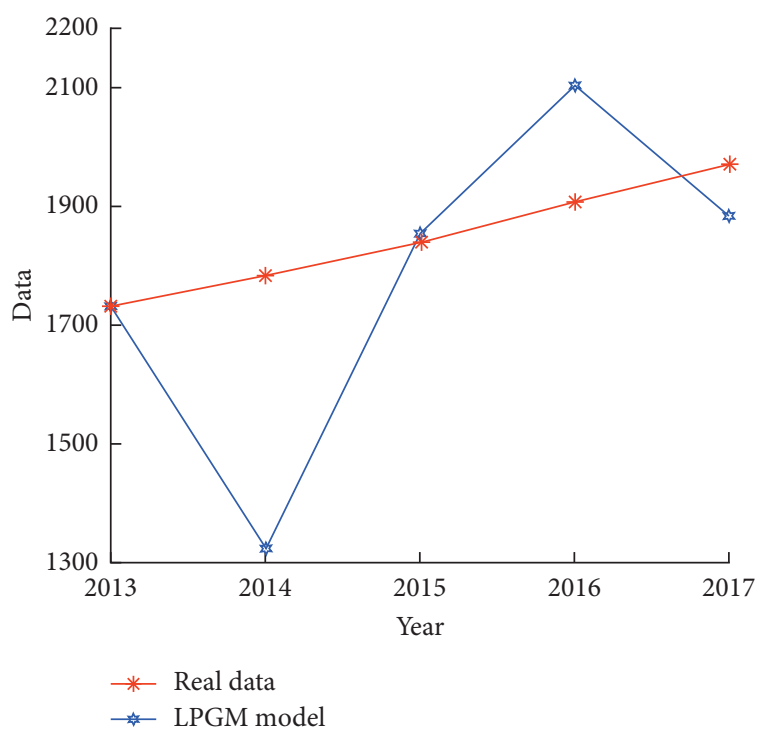

(a)

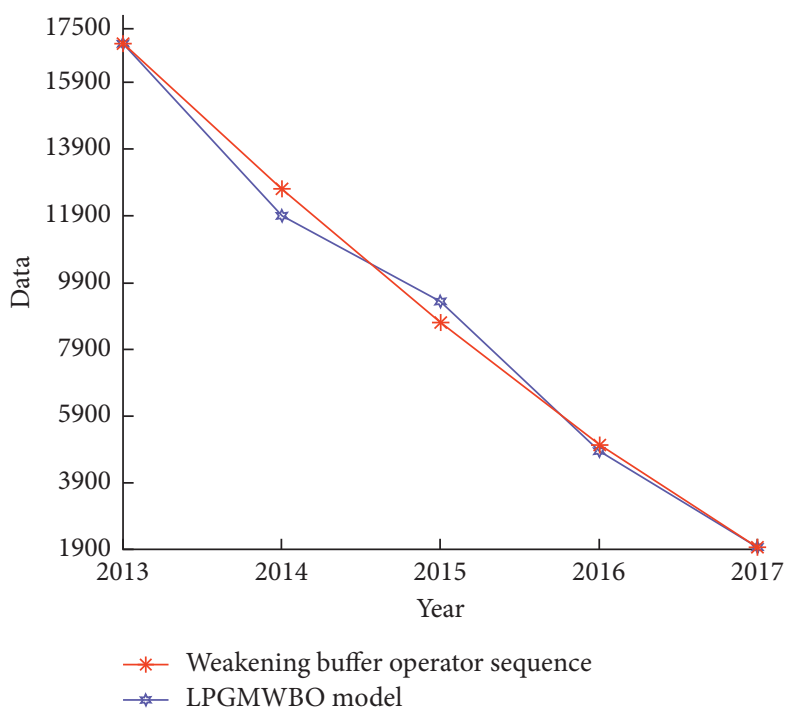

(b)

FIgURE 6: Simulated and forecasted data of the two models. (a) Simulated data of the LPGM $(1,1)$. (b) Simulated data of the LPGMWBO.

Table 12: Predicted urban population data for Chongqing, China, from 2018 to 2022 (unit: ten thousand).

\begin{tabular}{lccccc}
\hline Year & 2018 & 2019 & 2020 & 2021 & 2022 \\
\hline Urban population of Chongqing & 3277.63 & 7195.16 & 11333.84 & 11020.25 & 6685.68 \\
\hline
\end{tabular}

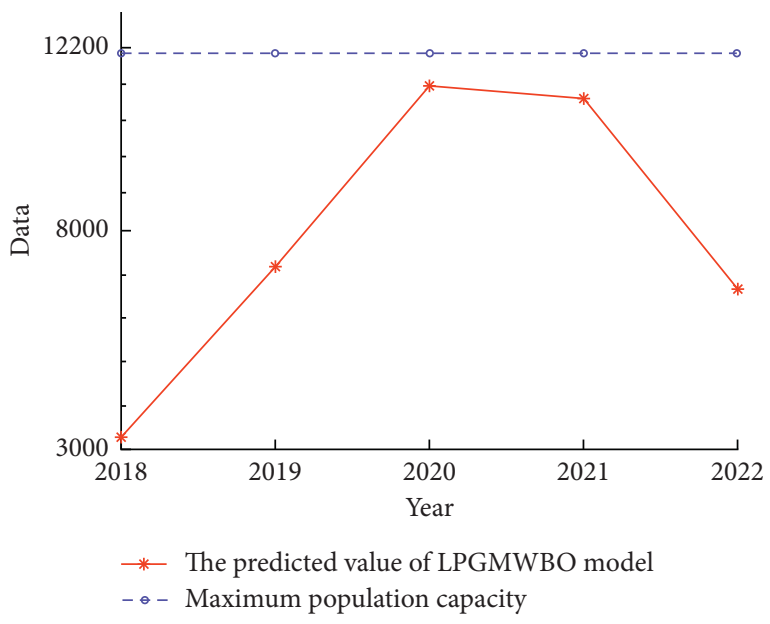

FIGURE 7: Population forecast trends for the urban population in Chongqing, China, 2018-2022. growth model. In addition, this result shows that the maximum capacity of the LPGM based on the logistic population growth model and the predicted population by the LPGMWBO based on the buffer operator are consistent with the ideal effect of the logistic population growth model. Therefore, the new LPGM and LPGMWBO are effective.

\section{Conclusion}

This paper starts with the logistic population growth model and combines the modeling mechanism of the grey prediction model to establish the LPGM. To further improve the accuracy of the model, a weakening buffer operator suitable for the power index is introduced to obtain the LPGMWBO; the maximum population capacity is estimated through the least-squares method, and the properties of the model are studied. The effectiveness of the LPGMWBO is 
demonstrated through six evaluation indexes of the three models in four practical cases.

The LPGMWBO not only solves the impractical situation in which the total population continuously increases, as in the Malthus model, but also solves problems such as the difficulty of obtaining a logistic solution and the possible distortion of the solution. Based on the empirical analysis, the MAPE of the LPGMWBO is approximately $4.5 \%$, and the accuracy level is between one and two. Furthermore, the trend of the prediction result curve meets the ideal effect of the logistic population growth model. Finally, the urban population of Chongqing is predicted to peak in 2020 and may decline in the following several years, which could help Chongqing formulate social strategies. Thus, this paper makes the following two contributions: this paper improves the classical population prediction model and effectively predicts the actual regional population.

However, changes in the population size are affected by many factors, such as society, economy, and the population itself, and the population situation changes over time. In this paper, the population can be effectively predicted only from the perspective of population change without considering the impact of other factors. Therefore, the changes in economic, social, environmental, and other factors and the relationship between these factors and population changes could be used to build a more accurate multivariate population prediction model that could not only address the deficiency of our current work but also enhance the prospects of our future work. This study could help the country forecast and control the population with scientific and accurate methods and provides a reliable scientific basis for the government in making strategic population decisions.

\section{Appendix}

In this appendix, we provide information on the Malthus population growth model and the logistic population growth model.

\section{A. Malthus Population Growth Model}

As early as the end of the 18th century, Thomas Malthus, a British demographer, studied more than a hundred years of demographic data and concluded that, in the process of natural population growth, the relative increase is a constant; the relative increase is the ratio of the population increase per unit of time to the total population at that time.

Let the total population be $N(t)$ at time $t$ and let the population growth rate (birth rate minus death rate) be $r$. According to Malthus's theory, in the process of natural population growth, $r$ is the increase in the population per unit of time and is directly proportional to the total population. Since the population growth continuously increases, it can be regarded as a differentiable continuous function. Therefore, the first model of the population increase is established as follows:

$$
\left\{\begin{array}{l}
\frac{\mathrm{d} N}{\mathrm{~d} t}=r N, \\
N\left(t_{0}\right)=N_{0} .
\end{array}\right.
$$

By separating the variables from the differential equation in (A.1), we can obtain the following:

$$
N(t)=N_{0} e^{r\left(t-t_{0}\right)}
$$

In this model, when $r>0$, the total population increases exponentially. In practice, population changes are examined annually. This model can also show whether the human living environment in a certain area is good, whether the human density is large, and, in the short term, whether the predicted and actual population statistics are mild; however, this approach is not suitable for predicting the actual population. Since $r>0$ and $t \longrightarrow+\infty$, this problem is infinite. Thus, the total population grows indefinitely, which is inconsistent with the actual population situation.

In fact, a key problem with the Malthus model, which is not consistent with reality, is the assumption that the natural growth rate of a population is a constant. On the contrary, this law is established only when the population is scarce and resources are abundant. When the growth reaches a certain degree and the total population reaches a certain base, negative factors hinder human development. For example, air pollution may occur with development. Other issues include environmental deterioration, the emergence of diseases that affect human lifespans, and adverse agricultural impacts that may lead to events such as food shortages and wars. Therefore, the Malthus model must be modified, and the following logistic population growth model is established.

\section{B. Logistic Population Growth Model}

In fact, there is a maximum population capacity, that is, $N_{m}$, in a region or an environment; thus, the growth rate in the Malthus model can be slightly altered. When $r>0$ and $t \longrightarrow+\infty$, the solution tends to a fixed constant, that is, $N_{m}$, which is called the environmental capacity. In addition, $\left(1-N / N_{m}\right)$ is the relative growth rate. It can be observed that the relative growth rate decreases as population $N$ increases. Assuming that $r$ is a quantity that is not affected by the environment and is only related to itself, it is called the intrinsic growth rate and is also known as the coefficient of life. When $N(t) \longrightarrow N_{m}$, the relative growth rate is 0 , and the total population tends to the limit value $N_{m}$. Therefore, the logistic growth model can be obtained as follows:

$$
\left\{\begin{array}{l}
\frac{\mathrm{d} N}{\mathrm{~d} t}=r N\left(1-\frac{N}{N_{m}}\right), \\
N\left(t_{0}\right)=N_{0} .
\end{array}\right.
$$

Formula (B.1) is a Bernoulli equation and can be solved as follows: 


$$
N(t)=\frac{N_{m}}{1+\left(N_{m} / N_{0}-1\right) e^{-r\left(t-t_{0}\right)}} .
$$

Based on formula (B.2), if $N_{0}$ and the total populations during two periods $N_{1}, N_{2}$ are known, we can obtain the following two equations:

$$
\left\{\begin{array}{l}
N_{1}=\frac{N_{m}}{1+\left(N_{m} / N_{0}-1\right) e^{-r\left(t-t_{0}\right)}} \\
N_{2}=\frac{N_{m}}{1+\left(N_{m} / N_{0}-1\right) e^{-r\left(t-t_{0}\right)}} .
\end{array}\right.
$$

The maximum population $N_{m}$ and the intrinsic growth rate $r$ can be obtained from the equations in formula (B.3) to predict the total population with the same spacing. This method uses only three types of data, which do not fully follow the law of population growth. Furthermore, this equation group is a nonlinear equation group, and analytically solving this group is difficult. There are many numerical solutions, and the results may be distorted. The growth model needs to be improved. The grey prediction model is simple and adaptable. This model can better address sudden changes in the parameters. Through data accumulation processing, the relationships in the data increase, the exponential relationship of a sequence increases, and the randomness of the data decreases. Then, the logistic parameters are estimated using the least-squares method. Consequently, a grey prediction model based on the logistic population growth model is established.

\section{Data Availability}

The data used to support the findings of this study are available from the corresponding author upon request.

\section{Conflicts of Interest}

The authors declare that they have no conflicts of interest.

\section{Acknowledgments}

This work was supported by Project of Humanities and Social Sciences Youth Fund of Ministry of Education of China (no. 19XJC630011), the Science and Technology Research Program of Chongqing Educational Committee (no. KJ1703057), the Humanities and Social Sciences Program of Chongqing Municipal Educational Committee (nos. 19SKGH043 and 18SKGH031), and the Chongqing Normal University Fund Project (no. 18XWB017).

\section{References}

[1] Yf. Tong, "Evolution, problems and prospect of population carrying capacity research," Population Research, vol. 36, no. 5, pp. 28-36, 2012.

[2] R. S. DeFries, T. Rudel, M. Uriarte, and M. Hansen, "Deforestation driven by urban population growth and agricultural trade in the twenty-first century," Nature Geoscience, vol. 3, no. 3, pp. 178-181, 2010.
[3] D. E. Bloom and R. B. Freeman, "The effects of rapid population growth on labor supply and employment in developing countries," Population and Development Review, vol. 12, no. 3, pp. 381-414, 1986.

[4] H. Zhong, "The impact of population aging on income inequality in developing countries: evidence from rural China," China Economic Review, vol. 22, no. 1, pp. 98-107, 2011.

[5] J. Q. Liu, "Analysis of Chinese population in twenty-first century," Population Study, vol. 33, no. 6, pp. 30-37, 2009.

[6] A. Wiśniowski, P. W. F. Smith, J. Bijak, J. Raymer, and J. J. Forster, "Bayesian population forecasting: extending the Lee-Carter method," Demography, vol. 52, no. 3, pp. 1035-1059, 2015.

[7] A. E. Raftery, N. Li, H. Sevcikova, P. Gerland, and G. K. Heilig, "Bayesian probabilistic population projections for all countries," Proceedings of the National Academy of Sciences, vol. 109, no. 35, pp. 13915-13921, 2012.

[8] H. L. Shang, P. W. F. Smith, J. Bijak, and A. Wiśniowski, "A multilevel functional data method for forecasting population, with an application to the United Kingdom," International Journal of Forecasting, vol. 32, no. 3, pp. 629-649, 2016.

[9] J. R. Bryant and P. J. Graham, "Bayesian demographic accounts: subnational population estimation using multiple data sources," Bayesian Analysis, vol. 8, no. 2, pp. 1-32, 2013.

[10] J. Bongaarts and R. A. Bulatao, Beyond Six Billion: Forecasting the World's Population, National Academy Press, Washington, DC, USA, 2000.

[11] R. J. Hyndman and H. Booth, "Stochastic population forecasts using functional data models for mortality, fertility and migration," International Journal of Forecasting, vol. 24, no. 3, pp. 323-342, 2008.

[12] Y. S. Li, "Model selection and parameter identification in population forecasting," Finance \& Economics, vol. 11, no. 2, pp. 24-28, 2004.

[13] C. Czado, A. Delwarde, and M. Denuit, "Bayesian Poisson logbilinear mortality projections," Insurance: Mathematics and Economics, vol. 36, no. 3, pp. 260-284, 2005.

[14] J. L. Deng, Estimate and Decision of Grey System, Huazhong University of Science and Technology Press, Wuhan, China, 2002.

[15] L. Kong and X. Ma, "Comparison study on the nonlinear parameter optimization of nonlinear grey Bernoulli model (NGBM $(1,1))$ between intelligent optimizers," Grey Systems: Theory and Application, vol. 8, no. 2, pp. 210-226, 2018.

[16] X. Ma, W. Q. Wu, Y. Y. Zhang et al., "Improved GM $(1,1)$ model based on Simpson formula and its applications," Journal of Grey System, vol. 31, no. 4, pp. 33-46, 2019.

[17] Z.-X. Wang and Q. Li, "Modelling the nonlinear relationship between $\mathrm{CO}_{2}$ emissions and economic growth using a PSO algorithm-based grey Verhulst model," Journal of Cleaner Production, vol. 207, pp. 214-224, 2019.

[18] B. Zeng, M. Tong, and X. Ma, "A new-structure grey Verhulst model: development and performance comparison," Applied Mathematical Modelling, vol. 81, no. 81, pp. 522-537, 2020.

[19] W. Q. Wu, X. Ma, Y. Wang, Y. Zhang, and Bo Zeng, "Research on a novel fractional GM $(\alpha, n)$ model and its applications," Grey Systems: Theory and Application, vol. 9, no. 3, pp. 356-373, 2019.

[20] H. M. Duan, X. P. Xiao, and Q. Z. Xiao, “An inertia grey discrete model and its application in short-term traffic flow prediction and state determination," Neural Computing and Applications, vol. 32, no. 12, pp. 8617-8633, 2020.

[21] X. Xiao, H. Duan, and J. Wen, "A novel car-following inertia gray model and its application in forecasting short-term traffic 
flow," Applied Mathematical Modelling, vol. 87, pp. 546-570, 2020.

[22] N. Xie and N. Chen, "Flexible job shop scheduling problem with interval grey processing time," Applied Soft Computing, vol. 70, pp. 513-524, 2018.

[23] Z.-X. Wang, D.-D. Li, and H.-H. Zheng, "Model comparison of GM $(1,1)$ and DGM $(1,1)$ based on Monte-Carlo simulation," Physica A: Statistical Mechanics and Its Applications, vol. 542, Article ID 123341, 2020.

[24] H. M. Duan and X. P. Xiao, "A multimode dynamic shortterm traffic flow grey prediction model of high-dimension tensors," Complexity, vol. 2019, Article ID 9162163, 18 pages, 2019.

[25] Y. Chen, W. Lifeng, L. Lianyi, and Z. Kai, "Fractional Hausdorff grey model and its properties," Chaos, Solitons \& Fractals, vol. 138, Article ID 109915, 2020.

[26] H. M. Duan, G. Y. Lei, and K. L. Shao, "Forecasting crude oil consumption in China using a grey prediction model with an optimal fractional-order accumulating operator," Complexity, vol. 2018, Article ID 3869619, 12 pages, 2018.

[27] Z. X. Wang and P. Y. Yao, "Grey relational analysis of economic policy uncertainty in selected European union countries," Economic Computation \& Economic Cybernetics Studies \& Research, vol. 52, no. 2, pp. 251-265, 2018.

[28] X. P. Xiao and H. M. Duan, "A new grey model for traffic flow mechanics," Engineering Applications of Artificial Intelligence, vol. 88, Article ID 103350, 2020.

[29] B. Zeng, H. Duan, and Y. Zhou, "A new multivariable grey prediction model with structure compatibility," Applied Mathematical Modelling, vol. 75, no. 75, pp. 385-397, 2019.

[30] Q. Z. Xiao, M. Y. Shan, M. Y. Gao et al., "Parameter optimization for Bernoulli model on biomass energy consumption prediction," Applied Soft Computing Journal, vol. 95, Article ID 106538, 2020.

[31] S. Mao, Y. Kang, Y. Zhang, X. Xiao, and H. Zhu, "Fractional grey model based on non-singular exponential kernel and its application in the prediction of electronic waste precious metal content," ISA Transactions, 2020.

[32] S. H. Mao, M. Zhu, X. P. Wang, and X. P. Xiao, "Grey Lotka Volterra model for the competition and cooperation between third party online payment systems and online banking in China," Applied Soft Computing, vol. 95, Article ID 106501, 2020.

[33] B. Zeng, M. Xin, and J. J. Shi, "Modeling method of the grey GM $(1,1)$ model with interval grey action quantity and its application," Complexity, vol. 2020, Article ID 6514236, 10 pages, 2020.

[34] H. Gao, T. Yao, and X. Kang, "Population forecast of Anhui province based on the GM $(1,1)$ model," Grey Systems: Theory and Application, vol. 7, no. 1, pp. 19-30, 2017. 